\title{
SOLVING SINGULAR CONVOLUTION EQUATIONS USING THE INVERSE FAST FOURIER TRANSFORM*
}

\author{
Eduard Krajník, Praha, Vincente Montesinos, Valencia, \\ Peter Zizler, Calgary, VÁclav Zizler, Praha
}

(Received August 30, 2010)

Abstract. The inverse Fast Fourier Transform is a common procedure to solve a convolution equation provided the transfer function has no zeros on the unit circle. In our paper we generalize this method to the case of a singular convolution equation and prove that if the transfer function is a trigonometric polynomial with simple zeros on the unit circle, then this method can be extended.

Keywords: singular convolution equations, fast Fourier transform, tempered distribution, polynomial transfer functions, simple zeros

MSC 2010: 42A85

\section{INTRODUCTION}

Consider a convolution equation

$$
a * g=f
$$

with $a$ and $f$ finite sequences ( $g$ can be an infinite two-sided sequence). Our task is to solve for the sequence $g$ given $a$ and $f$. Consider the Fourier Transform $\mathcal{F}$ yielding the following analogue in the Fourier domain

$$
\mathcal{F}(a) \mathcal{F}(g)=\mathcal{F}(f) .
$$

It is well known (Wiener's Lemma) that if $\mathcal{F}(a)$ has no zeros on the unit circle then

* The second author was supported in part by Proyecto MTM2008-03211, Ministerio de Ciencia e Innovación, by a grant BEST 2010-134 of the Generalitat Valenciana, and by a grant from the Universidad Politécnica de Valencia, PAID 2009, Spain. The fourth author was supported by a grant AVOZ 10190503 and IAA 100190901 (Czech Republic). 
the solution $g$ is unique (see, e.g., [5]). The inverse Fast Fourier Transform $\left(\mathrm{FFT}^{-1}\right)$ is a common procedure to solve for $g$ given $a$ and $f$. It is based on the following. We mesh up the unit circle, sample $\mathcal{F}(f) / \mathcal{F}(a)$ on this mesh, and then take the inverse Fast Fourier Transform. The finer the mesh is, the closer we are to the solution $g$. This method mimics the Riemann Sum Approximation for the continuous inverse Fourier Transform for each coefficient $g(n)$. A classical result on Riemann Sum Approximation for the Riemann integral of a continuous function stipulates that this pointwise convergence to the solution $g$ is at least $o(1 / n)$ (see, e.g., [8]).

In engineering applications this procedure is many times successfully applied also in the case where $\mathcal{F}(a)$ has zeros on the unit circle. However, this method would converge to a solution $g$ which no longer is unique. In this paper we define an extension of this method to the case of a singular convolution equation and prove some convergence results under certain but not too restrictive conditions. We will refer to this procedure as the FFT Inversion Method for (possibly) singular convolutions equations.

Let us sketch the needed background (see, e.g., [1], [2], and [6]). Let $\mathcal{S}$ denote the vector space of all rapidly decreasing (complex-valued) $C^{\infty}$-functions defined on $\mathbb{R}$, i.e., the set of functions $\varphi: \mathbb{R} \rightarrow \mathbb{C}$ such that $P \cdot D^{n} \varphi$ is a bounded function for every polynomial $P$ and every $n \in \mathbb{N} \cup\{0\}$ (here $D^{n}$ denotes the $n$th derivative operator). This space is endowed with a locally convex topology defined by the sequence of seminorms $\left\{q_{n}\right\}_{n \in \mathbb{N}}$ given by

$$
q_{n}(\varphi):=\max _{|k| \leqslant n} \max _{x \in \mathbb{R}}\left(1+|x|^{2}\right)^{n}\left|D^{k} \varphi(x)\right|, \quad \varphi \in \mathcal{S} .
$$

Then $\left(\mathbb{S},\left\{q_{n}\right\}_{n \in \mathbb{N} \cup\{0\}}\right)$ becomes a Fréchet locally convex space. Its dual, $\mathcal{S}^{\prime}$, is the space of tempered distributions, and we endow it with its $w^{*}$-topology, i.e., the topology on $\mathcal{S}^{\prime}$ of the pointwise convergence on the elements in $\mathcal{S}$.

The space $\mathcal{S}$ can be identified with a subspace of $\mathcal{C}_{\text {per }}^{(\infty)}(\mathbb{R})$, the space of all infinitely differentiable (complex-valued) $2 \pi$-periodic functions on $\mathbb{R}$ endowed with the locally convex topology inherited from the canonical topology on $\mathcal{C}^{(\infty)}(\mathbb{R})$ induced by a fundamental system of compacta. Precisely, to $\varphi \in \mathcal{S}$ we associate the mapping $\tilde{\varphi}:[0,2 \pi] \rightarrow \mathcal{C}$ given by

$$
\tilde{\varphi}(t):= \begin{cases}\varphi(\sigma(t)) & \text { if } t \in(0,2 \pi) \\ 0 & \text { otherwise }\end{cases}
$$

where $\sigma:(0,2 \pi) \rightarrow \mathbb{R}$ is defined as

$$
\sigma(t)=\frac{1}{2 \pi-t}-\frac{1}{t} \text { for all } t \in(0,2 \pi)
$$


The mapping $\varphi \mapsto \tilde{\varphi}$ is a linear homeomorphism from the space $\mathcal{S}$ into $C_{\text {per }}^{(\infty)}(\mathbb{R})$. As it is well known, the space $C_{\mathrm{per}}^{(\infty)}(\mathbb{R})$ and the space $s$ of rapidly decreasing sequences are linearly homeomorphic, when $s$ carries the topology defined by the system of seminorms $\left\{p_{n}\right\}_{n \in \mathbb{N}}$, where $p_{n}(a):=\sum_{k=1}^{\infty} n^{k}\left|a_{k}\right|, a=\left(a_{k}\right) \in s$ (see, e.g., [4, Theorem 2.10.H.8]). Recall that a sequence $\left\{a_{n}\right\}_{n \in \mathbb{Z}}$ is said to be rapidly decreasing if for all $r>0$ there exists $C=C(r)>0$ such that $\left|a_{n}\right| \leqslant C|n|^{-r}, n \in \mathbb{Z} \backslash\{0\}$. The sequence $\left\{b_{n}\right\}_{n \in \mathbb{Z}}$ is said to be slowly growing if there exists $r>0$ and $C>0$ such that $\left|b_{n}\right| \leqslant C|n|^{r}$ for all $n \in \mathbb{Z}$. The space of all the slowly growing sequences will be denoted by $\mathcal{M}$.

As it is well known, polynomially bounded functions define tempered distributions via integration, see e.g., [5]. Precisely, the following result holds.

Proposition 1. Let $g$ be a measurable function on $\mathbb{R}$ such that for some $m \in \mathbb{N}$, the function $\left(1+x^{2}\right)^{m} g(x)$ is bounded on $\mathbb{R}$. Then the map $F_{g}: \mathcal{S} \rightarrow \mathbb{C}$ defined as

$$
F_{g}(f)=\int_{\mathbb{R}} g(x) f(x) \mathrm{d} x, \quad f \in \mathcal{S},
$$

is a tempered distribution.

If there is no risk of misunderstanding, distributions $F_{g}$ defined via (2) will be denoted simply by $g$. Observe that every $\varphi \in \mathcal{S}$ can be considered, according to Proposition 1, as an element in $\mathcal{S}^{\prime}$.

A tempered distribution $F$ is said to be $(2 \pi)$-periodic if $\langle F, \varphi\rangle=\langle F, \psi\rangle$ whenever $\varphi \in \mathcal{S}$ and $\psi(x)=\varphi(x+2 \pi)$ for every $x \in \mathbb{R}$. In view of Proposition 1 , the function $e_{n}$ defined as $e_{n}(x)=\mathrm{e}^{\mathrm{i} n x}$ for $x \in \mathbb{R}$ defines a tempered distribution (denoted again by $e_{n}$ ) via $(2)$, and, obviously, $e_{n}$ is a periodic distribution. More generally, given a slowly growing sequence $a=\left\{a_{n}\right\}_{n \in \mathbb{Z}}$, the sequence $\left\{\sum_{n=-N}^{N} a_{n} e_{n}\right\}_{N \in \mathbb{N}}$ is $w^{*}$ convergent. Its $w^{*}$-limit will be denoted, accordingly, by $\sum_{n=-\infty}^{\infty} a_{n} e_{n}$, and it is again a periodic distribution, that will be denoted by $\mathcal{F}(a)$. If $\mathcal{P}$ denotes the subspace of $\mathcal{S}^{\prime}$ of all periodic distributions, then $\mathcal{F}$ maps $\mathcal{M}$ into $\mathcal{P}$. It is known, see [6] for example, that

$$
\mathcal{P}=\left\{\sum_{n=-\infty}^{\infty} a_{n} e_{n}:\left\{a_{n}\right\}_{n \in \mathbb{Z}} \text { a slowly growing sequence }\right\}
$$

so in fact $\mathcal{F}$ maps $\mathcal{M}$ onto $\mathcal{P}$ (see, e.g., [7]). 
We define the convolution $\left\{c_{n}\right\}_{n \in \mathbb{Z}}=c=a * b$ of an element $a=\left\{a_{n}\right\}_{n \in \mathbb{Z}} \in s$ and an element $b=\left\{b_{n}\right\}_{n \in \mathbb{Z}} \in \mathcal{M}$ by the formula

$$
c_{n}=\sum_{j=-\infty}^{\infty} a_{n-j} b_{j}, \quad n \in \mathbb{Z} .
$$

The following result has a simple proof, that will be omitted.

Proposition 2. Let $a \in s$ and $b \in \mathcal{M}$. Then $a * b$ is well defined and belongs to s. Moreover, $\mathcal{F}(a * b)=\mathcal{F}(a) \mathcal{F}(b)$.

R e m a r k. The conclusion of Proposition 2 needs some comments. It relates the distribution associated to a certain sequence to the product of two distributions. It is a problem to define properly in general the product of two distributions (see, e.g., [3]). However, if $F \in \mathcal{S}^{\prime}$ and $G \in \mathcal{S}$, the multiplication $F \cdot G$ is well defined (cf. op. cit.). If $a \in s$, then $\mathcal{F}(a)$ is a true function, and it belongs to $\mathcal{S}$. In our case (see below) we are interested in elements $a \in c_{00}$, i.e., sequences with finite support. In this case, convolution of $a$ with a (slowly growing) sequence $g$, and multiplication of $\mathcal{F}(a)$ with elements in $\mathcal{S}^{\prime}$ are, then, well defined.

\section{THE MAIN RESULT}

Consider, as above, the convolution equation

$$
a * g=f,
$$

with the assumption that $a$ and $f$ are finite sequences ( $g$ need not be finite). Assume now that $\mathcal{F}(a)$ (a $2 \pi$-periodic function on $\mathbb{R}$ that can be considered as a function on the unit circle $T$ ) has zeros on $T$ (we refer to this case by saying that the convolution equation $a * g=f$ is singular). Proposition 2 shows that the Fourier Transform $\mathcal{F}$ translates the convolution equation into

$$
\mathcal{F}(a) \mathcal{F}(g)=\mathcal{F}(f)
$$

Formally, $\mathcal{F}(g)=\mathcal{F}(f) / \mathcal{F}(a)$ (if it exists, it would be certainly a periodic tempered distribution, i.e., an element in $\mathcal{P}$ ), and the solution $g$ will be obtained just by applying $\mathcal{F}^{-1}$ to the former equality (the (generalized) FFT method). Obviously, this approach needs to be justified, since $\mathcal{F}(a)$, as a function, is not invertible. The inverse function $\mathcal{F}^{-1}: \mathcal{P} \rightarrow \mathcal{M}$ has a simple description, due to the orthogonality of 
the base $\left\{e_{n}\right\}_{n \in \mathbb{Z}}$ in $[0,2 \pi]$, where $e_{n}(x):=\mathrm{e}^{\mathrm{i} n x}$ for $x \in \mathbb{R}$ and $n \in \mathbb{Z}$. Precisely, if $F(x):=\sum_{n=-\infty}^{\infty} a_{n} \mathrm{e}^{\mathrm{i} n x}$ for $x \in \mathbb{R}$, then

$$
a_{n}=\frac{1}{2 \pi} \int_{0}^{2 \pi} F(x) e_{-n}(x) \mathrm{d} x \quad \text { for } n \in \mathbb{Z} .
$$

Note that this is not, strictly speaking, the action of the distribution $F$ on the function $e_{n}$, since, certainly, no $e_{n}$ belongs to $\mathcal{S}$.

The key observation to solve our problem is that whenever $\mathcal{F}(a)$ has only simple roots on the unit circle $T$, equation (5) still has a meaning when $F$ is replaced by $\mathcal{F}(f) / \mathcal{F}(a)$, at least in the Cauchy Principal Value sense, and that the sequence $\left(g_{n}\right)$ so obtained is a solution (among possibly an infinite number of solutions) of our convolution equation. This is the content (and the proof) of the following result.

Theorem 3. Consider a singular convolution equation $a * g=f$, with finite sequences $a$ and $f$, and assume that the transfer function $\mathcal{F}(a)$ has simple zeros on the unit circle. Then this convolution equation is solvable by the (generalized) FFT method and moreover the (non-unique) solution $g$ is a bounded (two-sided) sequence.

P r o o f. It is not difficult to see that, without loss of generality, we can reduce the problem to the case that $\mathcal{F}(a)=1-\mathrm{e}^{\mathrm{i} x}$ (i.e., when $a=(\ldots, 0,0,1,-1,0,0, \ldots)$, where 1 is in position 0 and -1 in position 1$)$. This is done by decomposing $\mathcal{F}(f) / \mathcal{F}(a)$ by using partial fractions and then scaling. We shall prove that, for every $n \in \mathbb{Z}$, the following integral exists in the sense of its Cauchy Principal Value:

$$
g_{n}:=(\mathrm{VP}) \frac{1}{2 \pi} \int_{0}^{2 \pi} \frac{\mathcal{F}(f)(x)}{1-\mathrm{e}^{\mathrm{i} x}} \mathrm{e}^{-\mathrm{i} n x} \mathrm{~d} x
$$

This is simple: Just observe that, for $m \in \mathbb{Z}$,

$$
\frac{\mathrm{e}^{\mathrm{i} m x}}{1-\mathrm{e}^{\mathrm{i} x}}=\frac{1}{2}\left[\left(\cos m x+\frac{\sin m x \sin x}{1-\cos x}\right)+\mathrm{i}\left(\sin m x-\frac{\cos m x \sin x}{1-\cos x}\right)\right] .
$$

The real part of $(7)$ is integrable in $[0,2 \pi]$ for all $m \in \mathbb{Z}$, and the imaginary part is antisymmetric with respect to $\pi$ (this follows easier by checking that the complex conjugate of $\mathrm{e}^{\mathrm{i} m(\pi+x)}\left(1-\mathrm{e}^{\mathrm{i}(\pi+x)}\right)^{-1}$ is $\left.\mathrm{e}^{\mathrm{i} m(\pi-x)}\left(1-\mathrm{e}^{\mathrm{i}(\pi-x)}\right)^{-1}\right)$. This gives the convergence of the integral in (6).

Let us check that the sequence $\left(g_{j}\right)_{j \in \mathbb{Z}}$ obtained in (6) satisfies the requirements: It is enough, as has been mentioned above, to take $f=\left(f_{n}\right)_{n \in \mathbb{Z}} \in c_{00}$ and $a=\left(a_{n}\right)_{n \in \mathbb{Z}}$, 
where $a_{0}=1, a_{1}=-1$, and $a_{n}=0$ otherwise. Then, for $n \in \mathbb{Z}$,

$$
\begin{aligned}
\sum_{j=-\infty}^{\infty} a_{n-j} g(j) & =a_{0} g_{n}+a_{1} g_{n-1}=g_{n}-g_{n-1} \\
& =(\mathrm{VP}) \frac{1}{2 \pi} \int_{0}^{2 \pi} \frac{\mathcal{F}(f)(x)}{1-\mathrm{e}^{\mathrm{i} x}} \mathrm{e}^{-\mathrm{i} n x} \mathrm{~d} x-(\mathrm{VP}) \frac{1}{2 \pi} \int_{0}^{2 \pi} \frac{\mathcal{F}(f)(x)}{1-\mathrm{e}^{\mathrm{i} x}} \mathrm{e}^{-\mathrm{i}(n-1) x} \mathrm{~d} x \\
& =(\mathrm{VP}) \frac{1}{2 \pi} \int_{0}^{2 \pi} \frac{\mathcal{F}(f)(x)}{1-\mathrm{e}^{\mathrm{i} x}} \mathrm{e}^{-\mathrm{i} n x}\left(1-\mathrm{e}^{\mathrm{i} x}\right) \mathrm{d} x \\
& =\frac{1}{2 \pi} \int_{0}^{2 \pi} \mathcal{F}(f)(x) \mathrm{e}^{-\mathrm{i} n x} \mathrm{~d} x=f_{n} .
\end{aligned}
$$

The sequence $\left(g_{n}\right)_{n \in \mathbb{Z}}$ is bounded. Indeed, recall that the Dirichlet kernel $D_{n}$, for $n \in \mathbb{N}$, is the function

$$
D_{n}(x)=\frac{\sin \left(n+\frac{1}{2}\right) x}{\sin \left(\frac{1}{2} x\right)} \text { for } x \in(0,2 \pi) \text {. }
$$

Note that

$$
\frac{\sin m x \sin x}{1-\cos x}=\frac{\sin m x}{\sin x}(1+\cos x) \text { for } x \in(0,2 \pi) \text {. }
$$

This shows, together with the fact $D_{n}$ satisfies $\int_{0}^{2 \pi} D_{n}(x) \mathrm{d} x=2 \pi$ for all $n \in \mathbb{N}$, that the real part in (7) remains bounded when $m \rightarrow \infty$.

Once the convergence of the integral (6) has been proved for all $n \in \mathbb{N}$, the computation is done by using the meshing procedure sketched in the Introduction. For this we rely on the FFT techniques, as usual.

Consider a mesh $\left\{x_{k}\right\}=\{2 \pi k / N\}_{k=0}^{N-1}$ of the interval $[0,2 \pi]$. Define

$$
\mathbf{u}_{M, N}(k)= \begin{cases}\frac{\mathcal{F}(f)\left(\mathrm{e}^{\mathrm{i} x_{k}}\right)}{\left(1-\mathrm{e}^{\mathrm{i} x_{k}}\right)} & \text { if }\left|\frac{\mathcal{F}(f)\left(\mathrm{e}^{\mathrm{i} x_{k}}\right)}{\left(1-\mathrm{e}^{\mathrm{i} x_{k}}\right)}\right|<M \\ 0 & \text { otherwise, }\end{cases}
$$

and note each $\mathbf{u}_{M, N}$ induces a tempered distribution given by the following action

$$
\left\langle\mathbf{u}_{M, N}, \varphi\right\rangle=\frac{1}{N} \sum_{j} \sum_{k} \mathbf{u}_{M, N}(k) \varphi\left(x_{k}+2 \pi j\right)
$$

for all $\varphi \in S$. Observe that

$$
\lim _{M, N \rightarrow \infty}\left\langle\mathbf{u}_{M, N}, \varphi\right\rangle=\langle\mathbf{u}, \varphi\rangle \quad \text { for all } \varphi \in S
$$


where $\mathbf{u}$ is the tempered distribution defined by the action

$$
\langle\mathbf{u}, \varphi\rangle=\int_{\mathbf{R}} \frac{\mathcal{F}(f)\left(\mathrm{e}^{\mathrm{i} x}\right)}{1-\mathrm{e}^{\mathrm{i} x}} \varphi(x) \mathrm{d} x .
$$

It follows that $\mathbf{u}_{M, N}$ converges to $\mathbf{u}$ as $M, N \rightarrow \infty$ in the $w^{*}$ topology. Let $g_{M, N}=$ $\operatorname{ifft}\left(\mathbf{u}_{M, N}\right)$ where ifft stands for the inverse Fast Fourier Transform. Therefore, there exists a bounded sequence $g \in M$ so that $g_{M, N} \rightarrow g$ coordinatewise.

Example I. We are solving

$$
a * g=f
$$

with the unit impulse $f=\left(f_{n}\right)_{n \in \mathbb{Z}}$, i.e., $f_{n}=0$ for all $n \in \mathbb{Z} \backslash\{0\}$ and $f_{0}=1$, and the mask $a=\left(a_{n}\right)_{n \in \mathbb{Z}}$ such that $a_{0}=a_{1}=a_{2}=1$ and $a_{n}=0$ otherwise. We thus have

$$
g_{n}+g_{n+1}+g_{n+2}=f_{n} \quad \text { for all } n \in \mathbb{N} .
$$

The corresponding transfer function

$$
\mathcal{F}(a)(z)=1+z+z^{2}
$$

has zeros $z=\mathrm{e}^{ \pm 2 \pi \mathrm{i} / 3}$ on the unit circle. Indeed, we have non-trivial solutions to the homogeneous equation

$$
g_{n}+g_{n+1}+g_{n+2}=\mathbf{0},
$$

for example

$$
\ldots,-1,-1,2,-1,-\mathbf{1}, 2,-1,-1,2, \ldots
$$

and so the solution is not unique.

We apply the FFT inversion method and obtain the sequence $g$ (using MATLAB):

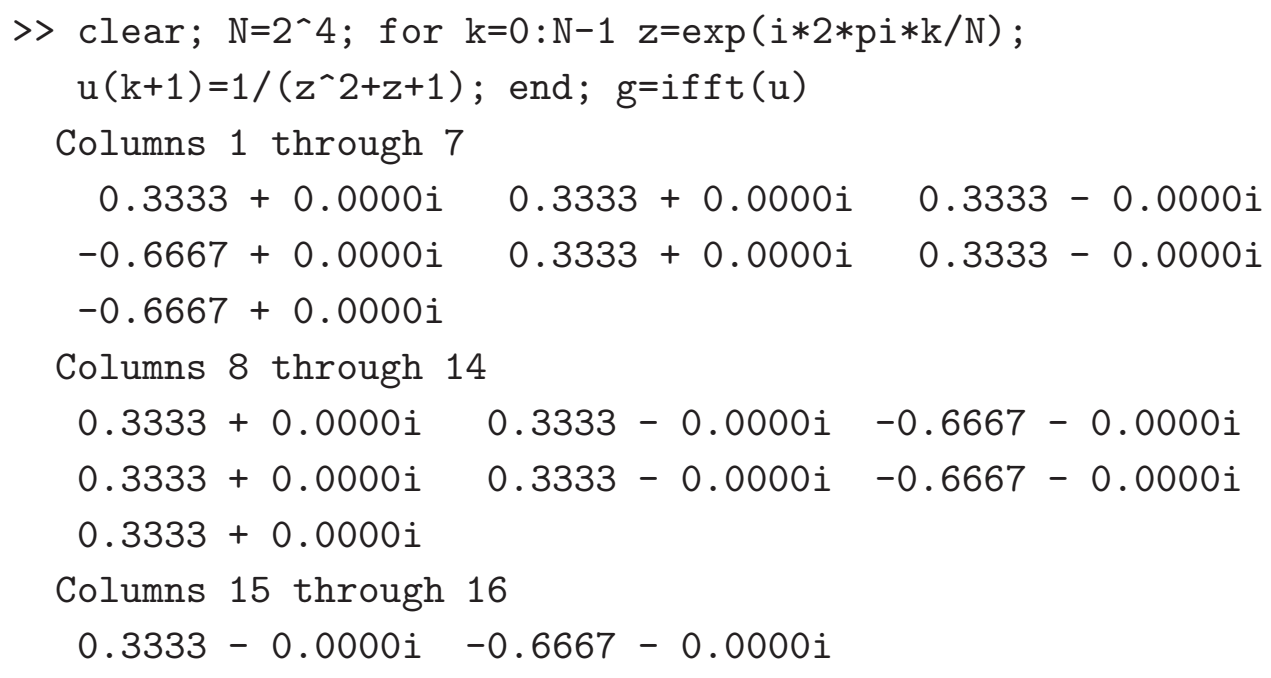


Re-grouping accordingly we get

$$
g=\ldots, \frac{1}{3}, \frac{1}{3},-\frac{2}{3}, \frac{1}{3}, \frac{1}{3},-\frac{2}{3}, \frac{\mathbf{1}}{\mathbf{3}}, \frac{1}{3}, \frac{1}{3},-\frac{2}{3}, \frac{1}{3}, \frac{1}{3},-\frac{2}{3}, \ldots
$$

Ex a m ple II. We are solving

$$
a * g=f
$$

with the unit impulse $f, f_{n}=0$ except $f_{0}=1$. We have the filter $a$ with $a_{0}=1$, $a_{1}=-2, a_{2}=1$ and $a_{n}=0$ otherwise. Our convolution equation becomes

$$
g_{n}-2 g_{n+1}+g_{n+2}=f_{n} .
$$

The corresponding polynomial transfer function

$$
a(z)=1-2 z+z^{2}=(z-1)^{2}
$$

has a multiple zero at $z=1$ on the unit circle. The FFT Inversion Method does not work in this case and

$$
\int_{0}^{2 \pi} \frac{1}{\left(1-e^{i x}\right)^{2}} d x
$$

fails to exist.

\section{References}

[1] I. Babuška: The Fourier transform in the theory of difference equations and its applications. Arch. Mech. 11 (1959), 349-381.

[2] R. Beals: Advanced Mathematical Analysis. GTM 12. Springer, New York-HeidelbergBerlin, 1973.

[3] B. Fisher: The product of distributions. Q. J. Math. 22 (1971), 291-298.

[4] H. Jarchow: Locally Convex Spaces. B. G. Teubner, Stuttgart, 1981.

[5] W. Rudin: Functional Analysis. McGraw-Hill, New York, 1973.

[6] E. Vitásek: Periodic distributions and discrete Fourier transforms. Pokroky mat., fyz. astronom. 54 (2009), 137-144. (In Czech.)

[7] G. G. Walter: Wavelets and Other Orthogonal Systems with Applications. CRC Press, Boca Raton, 1994.

[8] J. L. Walsh, W. E. Sewell: Note on degree of approximation to an integral by Riemann sums. Am. Math. Monthly 44 (1937), 155-160.

Authors' addresses: E. Krajnik, Department of Mathematics, Faculty of Eletrical Engineering, Czech Technical University in Prague, Technická 2, 16627 Prague 6, Czech Republic, e-mail: krajnik@math.feld.cvut.cz; V. Montesinos, Instituto de Matemática Pura y Aplicada, Universidad Politécnica de Valencia, C/ Vera, s/n. 46022 Valencia, Spain, e-mail: vmontesinos@mat.upv.es; P. Zizler, Department of Math. Physics and Engineering, Mount Royal University, 4825 Mount Royal Gate SW, Calgary, Alberta, Canada, e-mail: pzizler@mtroyal.ca; V. Zizler, Institute of Mathematics of the Czech Academy of Sciences of the Czech Republic, Žitná 25, 11567 Praha 1, Czech Republic, e-mail: zizler@math.cas.cz. 